\title{
La justice administrative de proximité au Cameroun
}

\author{
Olivier Fandjip*
}

\section{Résumé}

À la question de savoir si la justice administrative en Afrique francophone ne souffre plus des dysfonctionnements liés à son inégale répartition géographique, limitant ainsi son rapprochement des justiciables, la réponse doit être nuancée. Au Cameroun, avec notamment la nouvelle répartition spatiale des juridictions, la proximité du juge, l'une des conditions d'un meilleur accès à cette juridiction, n'est pas entièrement réorganisée. L'accès au juge reste difficile en raison, d'une part, des rapprochements insuffisants des juridictions inférieures, et, d'autre part, la centralisation de la juridiction d'appel. Au-delà du manque de proximité avec la juridiction d'appel, la proximité adoptée pour les juridictions inférieures ignore les difficultés liées aux moyens de transport et de communication. Cette architecture s'éloigne par exemple du modèle français. Le rapprochement des justiciables par les juridictions de l'ordre judiciaire, notamment dans les zones difficiles d'accès est une solution en visage able.

\section{INTRODUCTION}

En Afrique francophone, de manière générale, ${ }^{1}$ et au Cameroun en particulier, l'idée d'une justice de proximité semble être au centre des actions des pouvoirs publics. Elle se traduit certainement à travers les réformes récentes du système judiciaire. On peut rappeler les propos du ministre de la justice garde des sceaux. Il relève qu'au «Cameroun, entre réforme de la pratique administrative, amélioration du service rendu au public, investissements en tous genres, entres autres, aucun secteur de l'administration n'est épargné par ce vent de rapprochement de l'administration des administrés [...] afin non seulement d'instaurer le règne de plus de sérénité et de confiance entre administration et administrés, mais aussi de modifier l'attitude, le comportement et l'opinion qu'ont ces derniers de cette administration $[\ldots] »{ }^{2}$ Ainsi peut-on observer, poursuit l'auteur, « un souci compréhensible de rapprochement de la justice des populations non seulement d'un point de vue financier et procédural, mais aussi d'un point de vue géographique». ${ }^{3}$

* Enseignant-chercheur au grade d'assistant (université de Dschang-Cameroun), Dr en droit public, ancien boursier et Ater, membre du CMH, Université Clermont Auvergne (EA 4232-France).

1 Voir, par exemple : F. Hourquebie (dir.), Quel service public de la justice en Afrique francophone? Bruxelles, Bruylant, 2013, 216 p.

2 L. Esso, «Plus près... encore plus proche ... », Editorial, in, Justitia, Magazine d'informations générales du Ministère de la justice, ${ }^{\circ}$ 012, février 2019, p. 5.

3 Ibid. 
Ces propos conduisent à cerner, d'une part, l'intérêt de la notion de justice de proximité dans les États africains, notamment francophone, en général, et au Cameroun en particulier, et, d'autre part, d'en dégager une définition. Il s'agit là de deux éléments devant servir à la compréhension et à l'intérêt de la présente étude.

Sur la définition de la notion, on peut dire que la justice de proximité fait référence à une justice qui se veut proche des justiciables. Il s'agit, comme le soulignent certains auteurs, de la «proximité spatiale d'une justice physiquement [...] plus à la portée des justiciables; proximité humaine d'une justice plus soucieuse de compréhension, de négociation que de sanction; proximité temporelle, enfin, d'une justice qui, se voyant reprocher ses engorgements et ses lenteurs, cherche à y remédier $\gg{ }^{4}$ La proximité peut donc être géographique, humaine, procédurale et financière. La proximité peut aussi s'analyser, au-delà de l'aspect physique c'est-à-dire en fonction de ce que le juge paraît ou non "différent des autres acteurs sociaux, voire de se montrer sensible aux différents attraits sociaux, de manière à conserver le recul nécessaire au moment de juger $» .^{5}$

Dans le cas des pays d'Afrique francophone, en général, et du Cameroun en particulier, cette question a un intérêt singulier, et surtout du point de vue géographique. Cette particularité apparaît au regard des réformes récentes ayant notamment conduit, pour le cas du Cameroun, à la création de nouvelles juridictions administratives.

La doctrine a d'ailleurs eu l'occasion de se prononcer à ce sujet. Ainsi, par exemple, analysant l'ordre juridictionnel administratif des pays d'Afrique, le professeur Magloire Ondoa avait pu conclure, il y a quelques années, que «dans tous les cas [...] les justiciables africains, sans exception, malgré les assouplissements constatables ici ou là, demeurent éloignés de la juridiction [...] ». Ceci pour la simple raison que «dans leur écrasante majorité, les États attribuent [le contrôle juridictionnel de l'administration] aux plus hautes juridictions siégeant uniquement à la capitale». L'auteur concluait également que « la centralisation, source de distanciation géographique, apparaît au total comme l'effet le plus néfaste des politiques africaines de répartition spatiale de la compétence administrative $[\ldots] »{ }^{6}$ Ces propos semblent démentis, aujourd'hui, compte tenu, non seulement des réformes opérées mais aussi en raison des efforts d'aménagements du positionnement spatial des juridictions administratives dans de nombreux pays africains, en général, et au Cameroun, en particulier.

En effet, comme l'a relevé la doctrine en France, « la question de la distance à laquelle se tient la justice par rapport au justiciable constitue aujourd'hui l'une des principales thématiques qui servent d'enjeu, de guide, de critère, voire de topos, dans l'établissement des

4 A. Wyvekens, J. Faget, in J. Faget et al., La justice de proximité en Europe, Toulouse, Editions Eres, 2001, p. 8.

5 J. Fometeu, «La distance du juge. Chronique d'humeur à propos d'un dilemme de magistrat », $\mathrm{Ca}$ hiers juridiques et politiques, université de Ngaoundéré, numéro spécial : le juge et le droit, 2014, p. 105.

6 M. Ondoa, Le droit de la responsabilité publique dans les Etats en développement. Contribution à l'étude de l'originalité des droits africains postcoloniaux, Paris, L'Harmattan, 2010, p. 234. 
politiques judiciaires. Avoir une justice proche du justiciable, [...] des juges localisés à faible distance des usagers [...] semble s'imposer comme une nécessité pour garantir l'efficacité, la crédibilité, la pertinence ou la modernité de la pratique judiciaire $» .^{7}$ Cette affirmation rejoint celle du ministre camerounais en charge de la justice lorsqu'il écrit que, de manière concrète, le rapprochement entre la justice et les justiciables se manifeste par «l'enrichissement sans cesse de l'arsenal juridique, ainsi que par les multiples travaux de construction et de réhabilitation des infrastructures judiciaires $[\ldots] \gg{ }^{8}$

Pour bien cerner cette question, il faut dresser un état des lieux des évolutions de la justice administrative en Afrique francophone, en général, et camerounaise, en particulier. S'agissant des droits des pays d'Afrique francophone, ${ }^{9}$ de manière générale, parvenus à l'indépendance, ils ont opté pour des modèles de contentieux administratifs différents, sans remettre en cause le principe de la séparation des autorités administratives et judiciaires hérité du droit français.

En effet, qu'il s'agisse de l'adoption d'un système moniste, qui confie le contentieux administratif aux juridictions de droit commun, ou dualiste, avec la création des juridictions spéciales en matière administrative, les procédures applicables devant ces juridictions administratives sont restées particulières donc différentes de celles en vigueur devant les juridictions de droit commun. C'est ainsi, par exemple, que l'exigence du recours gracieux préalable représente l'un des éléments singuliers de la procédure devant ces juridictions.

On peut relever qu'en Afrique du Nord, le Maroc avait opté pour l'unité de contentieux avec une dualité dans son fonctionnement. Cette formule va être adoptée dans certains pays en Afrique au sud du Sahara. C'est le cas en Guinée, au Congo, au Togo, en Côte d'Ivoire, dans les Îles Comores, en Mauritanie et au Sénégal. Le Sénégal fut le premier pays à opter pour l'unité de juridiction suite à l'indépendance. Il faut rappeler que la justice administrative dans ces pays d'Afrique au sud du Sahara avant les indépendances était assurée par les Conseils du contentieux administratif installés dans l'ensemble de ces pays. Il en existait un pour l'Afrique occidentale française basé à Dakar au Sénégal et un autre pour l'Afrique équatoriale française situé à Brazzaville. ${ }^{10}$

7 B. Bastard, P. Guibentif, « Justice de proximité : la bonne distance, enjeu de politique judiciaire », Revue Droit et société, 2007/2, n 66, p. 267.

8 L. Esso, «Plus près... encore plus proche ... », op. cit, ibid.

9 Voir J. Vanderlinden, Aux sources des droits africains contemporains, Paris, Institut de droit comparé, 1975, p. 55 et 69.

10 En dehors de ces pays africains ralliés à la tradition juridique française, le modèle d'unité juridictionnel était en vigueur dans d'autres pays. C'était le cas au Ghana, en Guinée-Equatoriale, en Ile Maurice, en Guinée-Bissau, au Sao Tomé \& Principe, au Cap-Vert, au Mozambique. Ces pays appliquent essentiellement le système de Common-Law. Il y a aussi le cas des pays un peu proches du modèle français comme la Belgique qui appliquaient le système d'unité de juridiction en plein contentieux dans les pays comme le Rwanda, la République démocratique du Congo et le Burundi. Les pays qui initialement n'étaient pas ralliés à la tradition juridique française ont eu des penchants pour le dualisme juridictionnel. Il en est ainsi de la République démocratique du Congo qui, avec la Constitution du 18 février 2006, a opéré une subdivision de la Cour suprême de justice en Cour 
Il y a eu des changements intéressants concernant ces pays de tradition juridique francophone. En effet, on a observé des glissements allant du système moniste vers le dualisme juridictionnel. Ainsi, en Algérie, on a vu naître des Tribunaux administratifs notamment en 1998 à la suite de la mise en place deux années auparavant d'un Conseil d'État. Le cas le plus intéressant concerne le Maroc. Modèle d'unité de juridiction de référence parmi ces pays, le Maroc a mis en place en 1996 des Cours d'appel de l'ordre administratif. Déjà, cinq années auparavant, des Tribunaux administratifs avaient été créés notamment en 1991. ${ }^{11}$

De même, parmi les pays d'Afrique au sud du Sahara qui avaient opté pour le modèle d'unité de juridiction, certains se sont un peu penchés vers le modèle dualiste. C'est le cas du Sénégal qui avait, en 1992, mis en place un Conseil d'État avant de revenir à l'ancien modèle de Cour suprême encore en vigueur en matière d'excès de pouvoir. ${ }^{12}$ On peut aussi noter l'exemple de Djibouti, qui après avoir gardé le Conseil du contentieux administratif hérité de l'époque coloniale, l'a récemment, en 2009, remplacé par un Tribunal administratif juge de premier ressort en matière administrative.

D'autres pays, à l'opposé, ont opté pour un système dualiste. Il s'agit du Bénin, ${ }^{13} \mathrm{du}$ Mali, ${ }^{14}$ de la Tunisie,${ }^{15}$ de Madagascar, ${ }^{16}$ de la République centrafricaine, du Tchad, ${ }^{17} \mathrm{du}$ Gabon et surtout du Cameroun.

constitutionnelle, Conseil d'État et Cour de cassation (http://www.leganet.cd/Legislation/Droit\%2 0Judiciaire/OL.82.017.31.03.1982.htm, Consulté le 08 mars 2020 à 16 h). Désormais au Burundi, la Chambre administrative de la Cour suprême est chargée de juger en appel les décisions des juridictions inférieures en matière de responsabilité. Il s'agit des Cours administratives nouvellement mises sur pied (Loi du 14 janv. 1987 portant réforme du code de l'organisation et de la compétence judiciaire; loi du 25 févr. 2005 régissant la Cour suprême, art., 34, 37). Les pays comme la GuinéeBissau (Constitution du 16 mai 1984. Voirhttp://mjp.univ-perp.fr/constit/constitintro.htm. Constitutions du Monde), le Cap-Vert, n'appartenant pas eux aussi au modèle français, ont prévu qu'éventuellement des juridictions administratives pourraient être mises sur pied. Dans le premier cas, l'article 93 de la Constitution du 16 mai 1984 dispose que « l'existence des tribunaux exclusivement destinés au jugement d'une certaine catégorie d'infractions est interdite. Sont exceptés des dispositions du numéro précédent les tribunaux militaires dont la compétence s'exerce dans le jugement des infractions de même nature, qui pour des motifs pertinents leurs ont équivalents; les tribunaux administratifs, fiscaux et des comptes ». Au Cap Vert, l'alinéa 2 de l'article 228 de la Constitution dispose qu' « il peut exister des tribunaux de l'ordre judiciaire de seconde instance et des tribunaux administratifs » (Voir art. 222 et 228, Constitution du 14 févr. 1981, version du 4 sept. 1992. http://mjp.univ-perp.fr/constit/constitintro.htm. Constitutions du Monde).

11 A. S. Ould Bouboutt, « Le contentieux administratif comparé en France et dans les pays d'Afrique francophone », RDP, n 2/2013, p. 379.

12 http://www.gouv.sn/Loi-organique-portant-creation-de.html.

13 Loi n $^{\circ}$ 2001/37, 10 juin 2002, portant organisation judiciaire au Bénin.

14 Loi n $^{\circ}$ 61/55/AN/RM, 15 mai 1961, portant organisation judiciaire au mali.

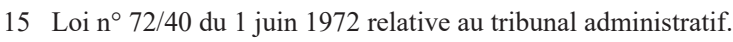

16 Voirhttp://madarevues.recherches.gov.mg/? Dix-ans-de-jurisprudence.

17 A. S. OuldBouboutt, « Le contentieux administratif comparé en France et dans les pays d'Afrique francophone », op. cit, ibid. 
En effet, dans la plupart de ces pays, la justice administrative était insérée au sein de la plus haute juridiction à savoir la Cour suprême dont la Chambre administrative connaissait en premier ressort le contentieux administratif à charge pour l'Assemblée plénière de statuer en appel contre les jugements rendus par ladite Chambre. Cela dit, les autres juridictions, notamment judiciaires, ne connaissent pas des matières administratives.

Dans ces systèmes également, des réformes ont été observées. C'est le cas en République centrafricaine où, selon l'alinéa 2 de l'article 85 de la Charte constitutionnelle du 18 juillet 2013, « la justice est rendue [...] par la Cour de cassation, la Cour des comptes, le Conseil d'État et les Cours et Tribunaux ${ }^{18}{ }^{18}$. C'est le cas également au Burkina Faso où la loi organique du 23 mai 2000 a mis en place un Conseil d'État chargé de connaître en appel les décisions rendues par les Tribunaux administratifs. Il s'agit, selon l'article $1^{\text {er }}$ de ce texte, de la «juridiction supérieure de l'ordre administratif». Des réformes sont également observées en Tunisie ${ }^{19}$ et à Madagascar. ${ }^{20}$

Ces évolutions relatives à la répartition spatiale de ces juridictions viennent donc apportées un bémol à l'idée de la proximité géographique desdites juridictions et notamment au Cameroun.

S'agissant du droit camerounais, en particulier, il faut souligner que depuis la Constitution camerounaise du 18 janvier $1996,{ }^{21}$ on peut relever que de nombreux changements sont intervenus, tant au niveau de l'organisation de la justice administrative ${ }^{22}$ qu'au niveau du système judiciaire ${ }^{23}$ en général. Cette loi fondamentale a offert des "perspectives $»^{24}$ à la justice administrative avec notamment la création des juridictions administratives infé-

18 Voirhttp://mjp.univ-perp.fr/constit/cf2013.htm.

19 Loi organique $\mathrm{n}^{\circ}$ 96/38, 30 juin 1996 relative à la répartition des compétences entre les Tribunaux judiciaires et le Tribunal administratif et à la création d'un Conseil des conflits de compétence.

20 Art. 121 de la Constitution du 11 déc. 2010.

21 Considérée comme une révision constitutionnelle pour les uns et nouvelle Constitution pour les autres, elle intervient avec l'amorce au cours de la décennie 90 du processus de démocratisation en Afrique; synonyme d'un contexte nouveau, caractérisé par l'apparition d'un certain nombre d'institutions opérant dans de nouvelles conditions, avec un accent mis sur la légalité, la transparence, la protection des droits fondamentaux et des libertés publiques. En ce sens, A. Ondoua (dir), La Constitution camerounaise du 18 janvier 1996. Bilan et perspectives, Yaoundé, Africaine d’Édition, 2007, $268 \mathrm{p}$.

22 M. P. Ze, « Réflexions sur la réforme de la justice administrative au Cameroun », Revue internationale de droit africain, $\mathrm{n}^{\circ}$ 89/2011, p. 57.

23 M. Kamto, «Les mutations de la justice à la lumière du développement constitutionnel de 1996 », Revue africaine des sciences juridiques, $\mathrm{n}^{\circ} 1 / 2000$, vol 1, pp. 9-20; J.-C. Aba'a Oyono, « Les mutations de la justice à la lumière du développement constitutionnel de 1996 », Juridis Périodique, n $\circ 44 / 2000$, pp. $74-85$.

24 C. Sietchoua Djuitchoko, «Perspectives ouvertes à la juridiction administrative du Cameroun par la loi n 96/06 du 18 janvier 1996 portant révision de la Constitution du 2 juin 1972 », Annales de la Faculté des Sciences Juridiques et Politiques de l'Université de Dschang, Yaoundé, Presses universitaires d'Afrique, t. 1, vol 1, 1997, pp. 162-175. 
rieures. ${ }^{25}$ L'on a observé, d'une part, la création des Tribunaux administratifs dans chaque région, et, d'autre part, l'érection de la Chambre administrative de la Cour suprême en juridiction d'appel et de cassation. C'est dire qu'il y a eu une rupture avec l'ancienne organisation qui faisait de l'appel la voie d'achèvement du procès administratif. ${ }^{26}$

La justice administrative camerounaise, entendue ici comme l'ensemble des Tribunaux chargés de connaître du contentieux des personnes publiques, et sans tenir compte des compétences exceptionnelles des Tribunaux judiciaires en la matière, est partie de l'ordonnance $n^{\circ} 72 / 06$ du 26 août 1972 relative à l'organisation et au fonctionnement de la Cour suprême dont la Chambre administrative, juge administratif, a été abrogée par la loi n 2006/016 du 29 décembre 2006 relative à l'organisation et au fonctionnement de la Cour suprême; ellemême modifiée en 2017. De même, la loi n 75/17 du 8 décembre 1975 fixant la procédure devant la Cour suprême statuant en matière administrative, a été remplacée par la loi $\mathrm{n}^{\circ}$ 2006/022 du 29 décembre 2006 relative à l'organisation et au fonctionnement des Tribunaux administratifs. Ces différentes dispositions se sont appuyées sur la Constitution du 18 janvier 1996 précitée. Le législateur l'a confirmé à travers la loi n 2006/015 du 29 décembre 2006 portant organisation judiciaire, modifiée par la loi n 2011/027 du 14 décembre 2011.

Sur le plan de la compétence matérielle, ces textes ont prévu que les Tribunaux administratifs connaissent de toutes les contestations dirigées contre les actes et décisions de l'administration, à l'exception des affaires attribuées spécialement par la loi à d'autres juridictions administratives. ${ }^{27}$ Ils examinent, notamment, les recours contre les actes de l'État, de ses démembrements, les actions en responsabilité dirigées contre les services publics administratifs et les dommages causés par l'activité des services publics, les contestations portant sur les contrats administratifs, le contentieux des élections locales. ${ }^{28}$

$25 \mathrm{Du}$ point de vue des textes en vigueur, soulignons qu'avant la restructuration, par la loi $\mathrm{n}^{\circ}$ 2006/016 du 29 décembre 2006, de la Cour suprême, organisée jadis par l'ordonnance n ${ }^{\circ} 72 / 06 \mathrm{du}$ 26 août 1972, l'ordonnance $n^{\circ} 72 / 04$ du 26 août 1972, portant organisation judiciaire, avait été au préalable remplacée par la loi n ${ }^{\circ}$ 2006/015 du 29 décembre 2006. Ce dernier texte consacra, lui aussi, les tribunaux administratifs (Tout comme l'article 42 alinéa 2 de la Constitution de 1996, révisée le 14 avril 2008, l'article 3 alinéa 3 de la loi n 2006/015, portant organisation judiciaire, consacre les tribunaux administratifs). Un autre aspect significatif, en cette matière, fût l'abrogation de la loi n ${ }^{\circ} 75 / 17$ du 8 décembre 1975 fixant la procédure devant la Cour suprême, statuant en matière administrative, par la loi $\mathrm{n}^{\circ} 2006 / 022$ du 29 décembre 2006 portant organisation et fonctionnement des tribunaux administratifs.

26 C. Sietchoua Djuitchoko, L'appel dans le contentieux administratif au Cameroun. Contribution à l'étude de la juridiction administrative, Thèse, Université d'Aix- Marseille III, 2001, Atelier national de reproduction de l'Université de Lille 3, $471 \mathrm{p}$.

27 Exemple de la Chambre administrative de la Cour suprême statuant en appel ou en cassation au Cameroun ou les Cours administratives d'appel et du Conseil d'État en France ou, d'une manière générale, les Tribunaux de droit commun. Pour ce dernier cas voir : article 3 de la loi n 2006/022, précitée.

28 Articles 2 alinéa 2, et, 14 alinéa 1 de la loi nº 2006/022. 
Comme on le voit, une nouvelle configuration a donc été adoptée. ${ }^{29}$ Ce renouveau de la justice administrative camerounaise ${ }^{30}$ est d'abord resté ineffectif. En effet, l'article 119 de la loi n 2006/022, susvisée, avait prévu, comme l'article 67 de la Constitution de 1996, que la mise en place effective des Tribunaux administratifs allait s'opérer « en fonction des besoins et des moyens de l'État ». Ces nouvelles institutions judiciaires allaient sans doute être mises en place de manière progressive. ${ }^{31}$ Le législateur n'avait apporté aucune précision, ni fixé une échéance relative à cette mise en place effective. C'est ainsi qu'à titre transitoire : d'une part, jusqu'à l'entrée en vigueur de la loi nº $2006 / 015$ du 29 décembre 2006 portant organisation judiciaire et de la loi n 2006/016 de la même année relative à l'organisation et au fonctionnement de la Cour suprême, c'est la Chambre administrative et l'Assemblée plénière de la Cour suprême qui statuaient en lieu et place de ces juridictions administratives créées par la Constitution de 1996. D'autre part, à la suite de la réforme, l'Assemblée plénière n'existait plus. La loi n 2006/016 précisait, en son article 142 alinéa 2 , que «les affaires pendantes devant l'ancienne assemblée plénière de la Cour suprême sur appel des jugements de la Chambre administrative sont transférées devant la section compétente de la Chambre administrative [...]». Surtout, l'article 140 disposait qu'en attendant la mise en place des juridictions inférieures, «les procédures antérieurement observées restent en vigueur ». L'article 141 alinéa 1 ajoutait que dès la mise en place de ces juridictions, «les affaires pendantes devant la Chambre administrative et relevant de la compétence de ces dernières leur sont transférées $»{ }^{32}$

Ainsi, ces Tribunaux administratifs restaient non opérationnels. Leur fonction était provisoirement confiée à la Chambre administrative de la Cour suprême. Autrement dit, d'un point de vue spatial, la justice administrative restait encore centralisée.

Dans un tel contexte, l'idée de proximité judiciaire ne pouvait pas être soutenue. Cette proximité judiciaire, entendue ici comme la possibilité pour le justiciable de recourir à un

29 Plus amplement, lire : P-E. Abane Engolo, Traité de contentieux administratif au Cameroun, Paris, L'Harmattan, 2019, 409 pages; O. Fandjip, Les mutations récentes de la justice administrative en Afrique francophone. Étude critique à partir du modèle camerounais, Paris, L'Harmattan, 2019, col. « Etudes africaines série droit », 250 p; O. Fandjip, « Les "citations directes" en droit camerounais du contentieux administratif », Jurisdoctoria, Université Paris I- Panthéon-Sorbonne, $\mathrm{n}^{\circ}$ 8/2012, pp. 131-156. Disponible sur le site : http://www.jurisdoctoria.net/numero8_juillet_2012.ht $\mathrm{ml}$.

30 M. P. Ze, « Réflexions sur la réforme de la justice administrative au Cameroun », op. cit,ibid; C. Keutcha Tchapnga, « La réforme attendue du contentieux administratif au Cameroun », Juridis Périodique, $\mathrm{n}^{\circ} 70 / 2007$, p. 25; C. Sietchoua Djuitchoko, «L'autonomie du recours en cassation en contentieux administratif au Cameroun (À propos de la réforme récente du contentieux administratif) », Juridis Périodique, n 82/2010, pp. 77-85 et n 83/2010, pp. 65-75; H.-C. Njocke, « Juridiction administrative : une réforme inachevée », Juridis Périodique, n 74/2008, pp. 49-63.

31 En ce sens, J. Owona, «L'essor du constitutionnalisme rédhibitoire en Afrique noire. Étude de quelques Constitutions Janus », Mélanges, Pierre-François Gonidec, Paris, LGDJ, 1985, p. 236.

32 B.-R. Guimdo Dongmo, « Le droit d'accès à la justice administrative au Cameroun. Contribution à l'étude d'un droit fondamental », RRJ, n 1/2008, p. 458. 
juge administratif qui, d'un point de vue géographique, lui est physiquement proche $^{33}$ n'était pas encore envisageable. Une telle situation conduisait donc encore à poser la question de l'accès au prétoire du juge administratif camerounais compte tenu de cet éloignement. La question de la proximité entre le juge et son justiciable devenait alors importante en rapport avec le droit d'accès au juge administratif. En effet, en droit interne, notamment au Cameroun, tout comme en droit international, ${ }^{34}$ le droit d'accès au juge suscite toujours des débats. ${ }^{35}$ A cette question se joint celle de la déconcentration de la justice car il existe un lien entre la déconcentration territoriale de la justice et l'accès à cette dernière ${ }^{36}$ donc avec celle de la proximité judiciaire. C'est certainement pour cette raison que, analysant la réforme judiciaire de 2006, le Doyen François Anoukaha relevait avec satisfaction la consécration d'une «nouvelle nomenclature des juridictions » avec comme apport majeur "le fait d'avoir intégré dans l'organisation judiciaire, les juridictions inférieures en matière de contentieux administratif ». Ainsi, poursuit l'auteur, ceci donnait " corps aux dispositions des articles 40 alinéa 3 et 41 alinéa 2 de la Constitution de $1996 » .{ }^{37}$

Mais il fallait attendre cinq années, à partir de la réforme de 2006, pour voir naître de manière effective cette réforme de la justice administrative. Cette naissance a été l'œuvre des décrets $n^{\circ} 2012 / 119^{38}$ et $194^{39}$ des 15 mars et 18 avril 2012 portant, respectivement, ouverture des Tribunaux administratifs et nomination des magistrats du siège dans lesdits Tribunaux. La création des Tribunaux administratifs telle que prévue par les lois $\mathrm{n}^{\circ}$

33 M. Charageat, « Marie Houllemare et Diane Roussel (dir.), Les justices locales et les justiciables. La proximité judiciaire en France du Moyen Âge à l'époque moderne », Criminocorpus [En ligne], Années antérieures, 2016, mis en ligne le 27 janvier 2016, consulté le 22 février 2020. URL : http:/ /journals.openedition.org/criminocorpus/3139.

34 Dans ce cas on note que ce droit est confronté aux immunités. Voir en ce sens : O. Fandjip, « Les immunités des organisations internationales et l'invasion des droits fondamentaux dans le droit international. Contribution à l'analyse des fondements et du sort des immunités des organisations internationales face à l'article $6 \S 1^{\text {er }}$ de la convention européenne des droits de l'homme dans la jurisprudence française et belge », $R R J, \mathrm{n}^{\circ} 2 / 2012, \mathrm{pp} .629-646$.

35 À cet égard, une doctrine récente préconise que l'accès au juge doit être codifié afin d'éviter l'arbitraire et les incompréhensions souvent à l'origine de nombre de rejet. Qu'en effet l'un des points les plus débattus, à propos de l'accessibilité à ce juge, est la qualité et l'intérêt pour agir; que parce que souvent restrictivement interprétés, ces points sont à l'origine de nombre d'irrecevabilités, ces deux conditions souffriraient de la trop grande tendance à céder aux règles en vigueur dans le droit privé. M. P. Ze, « Réflexions sur la réforme de la justice administrative au Cameroun », op. cit, pp. 66-67.

36 B.-R. Guimdo Dongmo, « Le droit d'accès à la justice administrative au Cameroun. Contribution à l'étude d'un droit fondamental », op. cit, p. 493.

37 F. Anoukaha, « La réforme de l'organisation judiciaire au Cameroun », Juridis Périodique n ${ }^{\circ} 68 / 2006$, p. 48.

38 Disponible sur le site de la présidence de la République du Cameroun : http://www.prc.cm/index_f r.php?link=les_actes/lecture_act\&id=400\&lang=fr\&quoi=Décret.

39 Disponible sur le site de la présidence de la République du Cameroun : http://www.prc.cm/index_f r.php?link=les_actes/lecture_act\&id=415\&lang=fr\&quoi=Décret,dernièreconsultation le 29 septembre 2017 à 18 h 49 min. 
2006/015 et 022, précitées, laissait apparaître de nombreuses interrogations auxquelles des réponses satisfaisantes ne pouvaient résulter que des dispositions conduisant à la mise en place concrète de cette déconcentration géographique de la justice administrative camerounaise. Ces deux décrets ont donc relancé la réflexion au sujet de la déconcentration de la justice administrative camerounaise, en général, en traitant en particulier des deux questions récurrentes suivantes : d'une part, l'accessibilité de la justice administrative; d'autre part, son indépendance au double plan organique et structurel. C'est le premier aspect à savoir celui du rapprochement physique de la justice administrative des justiciables qui est intéressant d'examiner ici. En effet, en dépit du fait que cette réforme a renforcé l'espoir de voir le Cameroun passer d'une "administration de souveraineté à une administration de gestion $»,{ }^{40}$ plus respectueuse des droits des usagers, il subsiste des interrogations sur la proximité physique entre le juge administratif et ses justiciables.

Il convient dès lors de se demander si la réforme du contentieux administratif traduitelle une déconcentration de la justice administrative susceptible de conforter la place du juge; de résoudre, véritablement, la question de l'éloignement géographique des juridictions longtemps déplorés dans le contexte camerounais. Autrement dit, la nouvelle répartition spatiale des juridictions administratives au Cameroun amène à se demander s'il est possible d'affirmer, désormais, que le justiciable camerounais se trouve proche de son juge, notamment sur le plan géographique, eu égard à la création des juridictions administratives déconcentrées. Cette problématique s'inscrit dans la suite de celle formulée récemment par un observateur. Selon ce dernier, dans le contentieux administratif camerounais aujourd'hui, la question centrale est celle de savoir si le juge administratif est désormais " effectivement capable d'instituer et de maintenir un équilibre entre les intérêts particuliers et l'intérêt général $\gg .{ }^{41}$

Mais pour en être véritablement apte, l'organisation de la justice administrative, notamment sa répartition spatiale, est un facteur important.

Pour répondre à cette question, il est nécessaire de dégager au préalable une hypothèse. Elle est la suivante : malgré les réformes observées au sein de la justice administrative dans les pays africains ralliés à la tradition juridique francophone, lesquelles visent à améliorer la qualité de la justice, notamment en la rapprochant davantage des justiciables, cette proximité demeure relative, notamment dans le cas du Cameroun, avec une centralisation de certaines juridictions.

Il est également important d'adopter une méthode de travail devant permettre de vérifier cette hypothèse. Le professeur Jean-Louis Bergel rappelle en ce sens que le droit est « inévitablement un système organisé autour d'un certain nombre de principes, de notions fondamentales, de procédés techniques dont la mise en ouvre suppose certaines mé-

40 C. Keutcha Tchapnga, «Les mutations récentes du droit administratif camerounais », Juridis Périodique, $\mathrm{n}^{\circ} 41 / 2000$, p. 80 .

41 C. Keutcha Tchapnga, Précis de contentieux administratif au Cameroun. Aspect de l'évolution récente, Paris, L'Harmattan, 2013, préface de Bernard-Raymond Guimdo Dongmo, p. 24. 
thodes $»{ }^{42}$ Cette idée rend compte de la nécessité de recourir à une méthode adaptée à l'étude du droit. Selon le professeur Jean Carbonnier, «toute science systématise; mais le droit, sous un certain aspect, semble n'être que cela. Les sources formelles font connaître les règles de droit dans la dispersion et parfois l'incohérence (même la synthèse que tente une codification peut n'être pas satisfaisante), avec des doubles emplois et, à l'inverse, des lacunes. Il faut classer, rassembler, compléter ou éventuellement éliminer, bref mettre en ordre, imprimer aux dispositions particulières l'unité d'un système ${ }^{43}{ }^{43}$ À cet égard, la systématisation et l'interprétation de la législation sont des moyens nécessaires en la matière. ${ }^{44}$

L'approche dogmatique ${ }^{45}$ permettra d'analyser, d'interpréter et de comprendre en quoi le rapprochement de la justice administrative des justiciables en Afrique francophone, notamment au Cameroun, reste mitigé compte tenu du maintien d'une certaine centralisation.

Aussi, pour y parvenir, on aura recours à ce que le professeur Jean Carbonnier a appelé les " sciences collatérales $d u$ droit ${ }^{46}$ à savoir la comparaison, l'histoire, ${ }^{47}$ la sociologie. Ce recours à une diversité de support permet de mieux étudier le droit. ${ }^{48}$ En effet, le professeur Jacques Chevallier fait observer que «les juristes ne peuvent se désintéresser des processus sociaux et politiques de production et d'application du droit [...], ils ne peuvent se désintéresser des valeurs sous-jacentes à l'ordre juridique et qui déterminent la légitimité de la norme juridique $»{ }^{49}$ Le professeur Jean Carbonnier soutient aussi cette approche. Selon lui, il faut mettre un bémol à l'approche soutenue par Hans Kelsen qui circonscrit le droit à un examen des normes, à une exégèse des textes. De son point de vue, «le droit

42 J.-L. Bergel, Théorie générale du droit, Paris, Dalloz, coll. « Méthodes du droit », $5^{\mathrm{e}}$ éd., 2012, p. 225. Voir aussi, M. Gravitz, Méthodes des sciences sociales, Paris, Dalloz, 10éd., 1996, p. 34.

43 J. Carbonier, Droit civil Introduction, Paris, PUF, coll. « Thémis droit privé », $27^{\complement}$ éd., 2002, p. 66.

44 Ibid.

45 La technique se distingue de la méthode dans la mesure où elle renvoie davantage à des opérations pratiques et non intellectuelles comme la méthode. On distingue ainsi la technique d'observation directe, ou documentaire, de celle dite indirecte portant par exemple sur des interviews. Elles n'ont pas été exclues dans la présente recherche. La méthode juridique comporte, d'une part, la dogmatique, et, d'autre part, la casuistique. Alors que la casuistique se focalise sur l'étude de l'application des normes et plus précisément sur la jurisprudence, c'est-à-dire l'étude des cas, l'approche dogmatique, quant à elle, est basée sur l'exégèse des textes, la systématisation du droit légiféré à partir des règles existantes pour en comprendre la cohérence ou les incohérences. En ce sens C. Atias, Épistémologie juridique, Paris, PUF, coll. « Droit fondamental », 1985, p. 91 et s.

46 J. Carbonier, Droit civil Introduction, op. cit., p. 71.

47 En ce sens J. Boudon, « La méthode juridique selon Adhémar Esmein », in N. Hakim et F. Melelleray (Études réunies par), Le renouveau de la doctrine française. Les grands auteurs de la pensée juridique au tournant du 20 siècle, Paris, Dalloz, coll. « Méthodes du droit », 2009, p. 263.

48 Dans le même sens, F. Ost, M. Van De Kerchove, « De la pluralité à la transversalité. La science juridique à la croisée des disciplines », in $\mathrm{C}$. Alonso et autres (dir), La pensée du doyen Hauriou à l'épreuve du temps, PUAM, 2015, p. 261.

49 J. Chevallier, «Pour une sociologie du droit constitutionnel », in L'architecture du droit, Mélanges en l'honneur de Michel Troper, Paris, Economica, 2006, p. 283. 
aspire à saisir, par-delà les textes, les phénomènes juridiques ${ }^{50}$ qui sont principalement des phénomènes collectifs et, ensuite, des phénomènes sociaux et individuels. Le droit « aspire à devenir [...] science de l'observation, voire de l'expérimentation ${ }^{51}{ }^{51} \mathrm{Il}$ convient donc de puiser également dans le «droit vivant », c'est-à-dire à adopter un positivisme sociologique. ${ }^{52}$ Partant de ces précisions d'ordre méthodologique, il sera question de démontrer que la mise en place effective d'une justice administrative spatialement proche des justiciables que l'on observe en Afrique, notamment au Cameroun, n'est que l'amorce d'une proximité (A). Il en est ainsi en raison des nombreuses insuffisances de cette délocalisation (B).

\section{A. L'amorce d'une justice de proximité par la déconcentration des juridictions administratives}

D'un point de vue géographique, la nouvelle organisation du contentieux administratif camerounais laisse apparaître une certaine proximité avec les justiciables. En effet, en vertu de la loi n 2006/022, le décret du 12 mars 2012, précité, a, d'une part, mis en place un Tribunal administratif dans chaque région (I). D'autre part, il a renforcé cette proximité spatiale au niveau de la fixation des ressorts respectifs desdits Tribunaux (II).

50 J. Carbonnier, Flexible droit, Pour une sociologie du droit sans rigueur, Paris, LGDJ, $10^{\mathrm{e}}$ éd., 2001, p. 257.

51 Ibid.

52 Ainsi, du point de vue de la démarche méthodologique, cette analyse de la justice administrative de proximité dans les pays africains, en général, et au Cameroun en particulier, reposera aussi sur la sociologie. Par ailleurs, la dimension historique ne sera pas exclue. L'étude du droit en Afrique de manière générale se doit d'accorder une place à l'histoire. Comme le rappelle Laboulaye, le droit, comme les études qui ont pour objet l'Homme et la société, doit être étudié en observant le passé, le présent et l'avenir. L'exclusion de ces aspects conduit vers une perception partielle donc incomplète des questions traitées. De même, s'agissant particulièrement du droit administratif et du droit constitutionnel, ces disciplines n'ont acquis le statut de « sciences véritables » qu'en étant fécondés par l'histoire et le droit comparé. En effet, puisque la science juridique est relative aux rapports des individus dans une société, elle est une matière pas très éloignée de l'anthropologie juridique et de l'histoire. Même si le juriste n'est pas un historien, il est impossible d'étudier sérieusement le droit administratif en ignorant la culture qu'elle véhicule et surtout l'histoire qui en est le résultat. D'ailleurs, l'histoire du droit administratif, particulièrement en France, fait partie des trois traits scientifiques qui le déterminent, à côté de son objet et de son mode de création. Voir F. Galletti, Les transformations de l'État et du droit public en Afrique de l'Ouest francophone, Thèse, Université de Perpignan, 2003, t. 1, p. 3; J. Boudon, « La méthode juridique selon Adhémar Esmein ", in N. Hakim et $\mathrm{F}$. Melelleray (Études réunies par), Le renouveau de la doctrine française... op. cit, p. 275; N. Rouland, L'anthropologie juridique, Paris, PUF, 1988, cité par J. Malaurie, « Droit et logique coloniale », in M. Doucet, J. Vanderlinden (dir), La réception des systèmes juridiques : implantation et destin, Bruxelles, Bruylant, 1994, p. 449; A. Delblond, Droit administratif, Bruxelles, Larcier, coll. « Manuel », 2009, p. 11. 


\section{Une justice de proximité liée à la création des Tribunaux administratifs régionaux}

L'analyse de la proximité judiciaire, s'agissant de la justice administrative au Cameroun (2), passe par un état des lieux de la carte judiciaire en vigueur (1).

1. L'état des lieux de la carte judiciaire au Cameroun

La carte judiciaire du Cameroun est certainement le reflet de la prise en compte du droit d'accès au juge. L'importance du droit d'accès au juge n'est plus à démontrer. Il suffit de rappeler qu'il s'agit d'un droit qui permet la réalisation d'autres droits. En effet, l'accès au juge peut s'entendre comme le droit pour chaque citoyen "de se faire rendre justice " devant une juridiction impartiale et équitable. Il correspond à la dimension organique de l'accès au droit, à côté de sa dimension normative, ${ }^{53}$ c'est-à-dire du droit d'accéder aux normes. Ce droit est consacré au préambule de la Constitution camerounaise de 1996 qui dispose que : «la loi assure à tous les hommes le droit de se faire rendre justice ». Il apparait également dans la Charte africaine des droits de l'Homme et des peuples, en son article 7, qui dispose que : «toute personne a droit à ce que sa cause soit entendue. Ce droit comprend: a) le droit de saisir les juridictions nationales compétentes de tout acte violant les droits fondamentaux qui lui sont reconnus et garantis par les conventions, les lois, les règlements et coutumes en vigueur; b) le droit à la présomption d'innocence, jusqu'à ce que sa culpabilité soit établie par une juridiction compétente; c) le droit à la défense, y compris celui de se faire assister par un défenseur de son choix; d) le droit d'être jugé dans un délai raisonnable par une juridiction impartiale ». C'est certainement en vertu de l'importance de ce droit, ainsi que de ces différentes dispositions, adoptées tant au plan national qu'international en la matière, que le législateur camerounais n'a de cesse d'améliorer la carte judiciaire.

En effet, selon les dernières références diffusées par les services du ministère de la justice, la carte judiciaire du Cameroun se présente de la manière suivante :

- une Cour suprême

- un Tribunal criminel spécial

- dix Cours d'appel

- dix Tribunaux administratifs

- douze Tribunaux de grande instance

- quarante-six Tribunaux de première et grande instance

- vingt-neuf Tribunaux de première instance

- un Parquet général de la Cour suprême

- un Parquet général du tribunal criminel spécial

- dix Parquets généraux des cours d'appel

53 En ce sens, V. Donier, B. Laperou-Scheneider (dir.), L'accès au juge, Recherches sur l'effectivité d'un droit, Bruxelles, Bruylant, 2013; X. Souvignet «L'accès au droit, Principe du droit, principe de droit », Jurisdoctoria, n 1/2008, p. 23 et 26. 
- trois Parquets d'instances autonomes

- quarante Parquets d'instances

- $\quad$ vingt Parquets autonomes. ${ }^{54}$

Il ressort du Plan national de promotion et de protection des droits de l'Homme au Cameroun que cette carte judiciaire s'aligne de manière progressive sur la carte administrative du pays avec notamment «la création de nouveaux Tribunaux de première et de grande instance » ainsi que «l'ouverture et la mise en place des Tribunaux administratifs ». ${ }^{55}$

Cette répartition est sans doute la réponse des pouvoirs publics aux critiques récurrentes adressées à la justice, notamment sur sa répartition géographique. Ainsi que le souligne le ministre de la justice, face à ces critiques, le gouvernement « a entrepris depuis plusieurs années de redorer l'image de la justice, garante de toute légitimité et surtout d'équité sociale». Cela s'est traduit, par exemple, par «les réponses adéquates apportées aux revendications exprimées par certains avocats anglophones, relatives au système judiciaire en vigueur [...], à l'accès au droit, à l'organisation et au fonctionnement général du système judiciaire et le vaste chantier engagé dans la modernisation des infrastructures judiciaires ». Ainsi, précise également le garde des sceaux, « le vent qui souffle sur la chancellerie et les juridictions est un vent de rénovation et d'innovations ». Parlant de la rénovation, il souligne qu'il s'agit de "dépoussiérer ce qui existe déjà et qui a pris un coup de vieux », de " donner un éclat nouveau et rompre avec certaines habitudes ». ${ }^{56}$ Quant à l'innovation, il est question de mettre la justice dans les conditions devant lui permettre de répondre, d'une part, aux attentes des populations, et, d'autre part, aux « standards internationaux $» .{ }^{57} \mathrm{C}$ 'est donc dans le cadre de cette répartition que s'insèrent les Tribunaux administratifs régionaux caractérisés par une certaine proximité avec les justiciables.

2. La justice de proximité par l'insertion des Tribunaux administratifs régionaux dans la carte judiciaire

La carte judiciaire du Cameroun s'est enrichie en 2012 par la naissance effective des Tribunaux administratifs. L'étude du décret du 15 mars 2012 portant création des Tribunaux administratifs, notamment en son article premier, permet d'identifier, d'une part, les différentes régions du Cameroun qui ont été dotées d'un Tribunal administratif, et, d'autre part, les appellations qui vont avec.

$54 \mathrm{http} / / /$ minjustice.gov.cm/index.php/fr/38-index-categories-fr/carte-judiciaire-et-penitentiaire?layou $\mathrm{t}=\mathrm{blog}$. Consulté le 04 octobre 2020.

55 Plan d'action national de promotion et de protection des droits de l'Homme au Cameroun (20152019), p. 27. Disponible sur le site : http://minjustice.gov.cm/index.php/fr/missions/droits-de-1-ho mme. Consulté le 04 nov. 2020.

56 L. Esso, «Un vent de changement... », Editorial, in, Justitia, Magazine d'informations générales du Ministère de la justice, $n^{\circ}$ 010, décembre 2017, p. 5.

57 Ibid. 
Sur la désignation de ces unités administratives dotées d'un Tribunal administratif, l'article premier de ce texte dispose que dans les régions ci-dessous indiquées, à savoir : les régions de l'Adamaoua, du Centre, de l'Est, de l'Extrême-Nord, du Littoral, du Nord, du Nord-Ouest, de l'Ouest, du Sud et du Sud-ouest, sont ouverts des Tribunaux administratifs. On note à ce niveau la mise en place d' « un Tribunal administratif par région » prévue par l'article 5 alinéa 1 (i) de la loi $n^{\circ} 2006 / 022$ portant organisation et fonctionnement des Tribunaux administratifs. Une logique presque similaire s'observe en ce qui concerne leurs appellations respectives.

Du point de vue de l'étiquette attribuée à chacun de ces Tribunaux, le législateur n'a eu d'autre repère que les appellations correspondantes à chaque chef-lieu de région suivant le découpage des circonscriptions administratives en vigueur. En effet, conformément à l'article 61 alinéa 1 de la Constitution, sont constituées en régions les dix provinces actuelles du pays. L'article 4 du décret $n^{\circ}$ 2008/376 du 12 novembre 2008, portant organisation administrative de la République du Cameroun, reprend ce découpage. Le Tribunal administratif de la région du Centre, à titre d'exemple, est désigné par : "Tribunal administratif de Yaoundé».

Le législateur n’a cependant pas considéré certaines dispositions de la loi n 2006/022, précitée, notamment l'article 5 alinéa 2 . En effet, cet article dispose que : «toutefois, suivant les nécessités de service, le ressort d'un Tribunal administratif peut être, par décret du président de la République, étendu à plusieurs régions ». Une formule similaire est d'ailleurs adoptée par l'article 19 alinéa 1 (ii) de la loi n²006/015 du 29 décembre 2006, portant organisation judiciaire, notamment en ce qui concerne les Cours d'appel des juridictions de l'ordre judiciaire. On pouvait alors, face à une telle disposition, se poser la question de savoir si en cas d'adoption d'une telle solution, quelle devait être la dénomination à attribuer à untel Tribunal administratif? Allait-on lui attribuer les dénominations des différentes régions dont il couvre les ressorts, respectifs, ou alors celle d'une seule d'entre elles? À titre d'exemple, comment allait-on dénommer le Tribunal administratif couvrant à la fois les régions du Nord, de l'Adamaoua et de l'Extrême-Nord? Comme solution, face notamment au silence du législateur, la suggestion faite par un observateur, et à laquelle il est intéressant de souscrire, peut être adoptée. En effet, celui-ci proposait de qualifier de telles juridictions de "Tribunaux interrégionaux »; expression qui semble adéquate à la situation résultant d'une extension du ressort d'un Tribunal administratif au ressort de plusieurs autres régions. ${ }^{58}$

Pour ce qui est des appellations qui correspondent à chacune des juridictions administratives, on devra se référer à la colonne numéro 2 du tableau dressé à l'article 2 du décret

58 C. Sietchoua Djuitchoko, « La difficile gestation des juridictions inférieures des comptes au Cameroun », Juridis Périodique n 68/2006, p. 101. En France également, le décret-loi de 1926 avait supprimé certains conseils de préfecture et attribua un caractère interdépartemental à ceux qui étaient maintenus lesquels allaient devenir, en 1953, des Tribunaux administratifs. En ce sens, J. Devaux, «La réforme des conseils de préfecture », RDP, 1926, p. 647; J. Rivero, « La réforme du contentieux administratif », RDP, 1953, p. 926. 
du 15 mars 2012. Ce tableau fixe à la fois les sièges et les ressorts des différents Tribunaux administratifs. Ainsi, la création des Tribunaux administratifs dans chaque région du pays conduit à un rapprochement de cette justice des citoyens dans la mesure où, auparavant, les justiciables devaient se rendre uniquement à Yaoundé pour saisir la juridiction administrative. La composition de ces Tribunaux administratifs est également intéressante à préciser. En effet, ceux-ci sont composés, au siège, de :

- un président;

- des juges;

- un greffier en chef;

- de greffiers.

Cet organe est chargé de rendre les décisions au sein desdites juridictions. En effet, comme son nom l'indique, la formation de jugement rend des décisions techniquement appelées des jugements. La loi a prévu que les jugements doivent être rendus après délibération, à la majorité des voix des juges ayant suivi les débats. En dehors des jugements, la formation de jugement peut aussi rendre des ordonnances. Celles-ci relèvent de l'office ou alors de la juridiction du président du Tribunal administratif ou le magistrat qu'il délègue à cet effet. Ainsi en sera-t-il en matière de référé et de sursis à exécution.

L'organisation de ces Tribunaux au niveau du parquet, encore dénommé ministère public, est différente. Au niveau du parquet, il est composé de :

- du procureur général près la Cour d'appel du ressort du Tribunal administratif;

- un ou plusieurs substituts du procureur général.

Il s'agit du ministère public, organe qui veille à la bonne application des lois et des règlements. En effet, comme le prévoit l'alinéa 1 de l'article 29 de la loi n 2006/015 du 29 décembre 2006 portant organisation judiciaire, modifiée en 2011, « le ministère public ou parquet veille à l'application des lois, règlements et décisions de justice. Il peut, dans l'intérêt de la loi, prendre devant toute juridiction auprès de laquelle il est représenté, les réquisitions qu'il estime utiles ». Comme dans le cas de la Chambre administrative de la Cour suprême, des juges non magistrats ont aussi été prévus au sein de ces Tribunaux administratifs. Il s'agit des juges ou substituts non magistrats à savoir :

- les professeurs de droit;

- les chargés de cours de droit;

- les fonctionnaires de catégories « $A$ » et cadres contractuels. Ceux-ci pourront être membres des Tribunaux administratifs en service extraordinaire pour un mandat de cinq ans.

Le législateur précise les conditions d'admissibilité à ces postes. Cela traduit un souci de rigueur et de spécialisation car la gestion du contentieux de l'administration exige des juges une connaissance importante du droit administratif et de l'administration. C'est pourquoi, pour les professeurs, peuvent être nommés, ceux ayant exercé pendant dix années consécu- 
tives, quinze ans pour les chargés de cours, et, enfin, pour la dernière catégorie, ceux-ci doivent être titulaire d'une maîtrise en droit et justifier de quinze années de fonction.

Après la répartition des Tribunaux administratifs, le législateur, plus précisément le pouvoir réglementaire, et conformément à la loi, a songé à désigner les membres de ces différents Tribunaux administratifs. Le décret du 18 avril 2012 organise donc la composition au siège de ces Tribunaux. À cet effet, le décret du 18 avril 2012 a désigné trois magistrats aux sièges des différents Tribunaux parmi lesquels un président comme l'indique l'article 6 alinéa 1 a (i) et (ii) de la loi n 2006/022 susvisée. Il en est ainsi parce que le Tribunal administratif siège en formation collégiale de trois membres et leurs décisions sont rendues à la majorité des voix. Par ailleurs, le président peut être suppléé conformément au texte portant statut de la magistrature en cas d'absence ou d'empêchement. ${ }^{59}$

Lorsqu'on confronte le décret du 18 avril 2012 à certaines dispositions de la loi $\mathrm{n}^{\circ}$ 2006/022, des doutes surviennent. " A priori », cette composition paraît insuffisante parce que le texte ne désigne ni les personnels du service des greffes ni les juges non magistrats. Pour ce qui est de l'absence des juges non magistrats, le décret du 18 avril 2012 ne semble pas avoir fait une erreur parce que : d'une part, l'article 6 de la loi $n^{\circ} 2006 / 022$ qui fixe la composition du Tribunal administratif ne fait pas allusion à cela et, d'autre part, parce que l'article 8 alinéa 2 de la loi ci-dessus visée n'en fait pas un impératif. Il dispose que cette catégorie de juge ou substitut en service extraordinaire au sein des Tribunaux administratifs peut être nommée pour les besoins du service. Le législateur n'a donc pas jugé nécessaire d'en désigner pour le moment.

Toutefois, leur désignation aurait été aussi l'occasion d'apporter plus de précisions sur leur statut. ${ }^{60}$

Au sujet des greffiers en chef et les greffiers, ${ }^{61}$ le législateur n'en fait, sans doute, pas mention dans le décret du 18 avril 2012 parce que le greffier en chef et les greffiers du Tribunal administratif sont nommés conformément au texte portant statut des personnels des greffes et à celui portant organisation administrative des juridictions. ${ }^{62}$

Cela dit, cette mise en place des Tribunaux administratifs, de manière générale, s'inscrit, comme le souligne le ministre de la justice, dans le cadre d'un « arrimage des cartes judiciaire et pénitentiaire à la carte administrative ». Cet arrimage "participe de [l'] élan de rapprochement de la justice du justiciable », il apparaît également comme étant « une preuve, s'il en était encore besoin, de ce que la distance judiciaire qui s'était créée entre justice et justiciable du fait des longues distances géographiques à parcourir par les justiciables pour avoir accès à la justice et tous les désagréments à elles imputées ${ }^{63}$ tendent à

59 Article 13 de la loi ${ }^{\circ}$ 2006/022.

60 C. Keutcha Tchapnga, «La réforme attendue du contentieux administratif au Cameroun », op. cit, p. 27.

61 Prévus par l'article 6 alinéa 1 a (iii) et (iv).

62 Article 11 de la loi n 2006/022, précitée.

63 L. Esso, «Plus près... encore plus proche... », Editorial, in, Justitia, Magazine d'informations générales du Ministère de la justice, $\mathrm{n}^{\circ} 012$, février 2019, ibid. 
disparaître.Dans le cadre de cette proximité judiciaire, il convient de remarquer que le législateur a innové en ce qui concerne la détermination des sièges et ressorts des différents Tribunaux administratifs créés. Cela contribue également à cette proximité physique entre le juge administratif et les justiciables.

\section{Une justice de proximité dans la fixation des sièges et ressorts des Tribunaux administratifs régionaux}

La répartition des sièges et des ressorts des Tribunaux administratifs régionaux est précisée dans les colonnes numéro trois et quatre du tableau dressé à l'article 2 du décret du 15 mars 2012 précité.

Sur les sièges desdites juridictions, l'article 2 du décret désigne, notamment à la colonne numéro trois, les chefs-lieux des différentes régions comme sièges des différents Tribunaux administratifs. En effet, il y a une coïncidence entre l'appellation attribuée à chaque Tribunal administratif et le siège de ladite juridiction. Le Tribunal administratif de Douala, région du Littoral, a son siège à Douala. Il faut observer qu'il y a une référence à la loi $\mathrm{n}^{\circ}$ 2006/022, notamment en son article 5 alinéa 1 (ii) qui fixe les sièges desdites juridictions dans les chefs-lieux des régions.

Pour ce qui est des ressorts de ces Tribunaux, le décret du 15 mars 2012 a certainement innové. L'innovation est liée au fait que ce texte a désigné, face au silence de la loi $\mathrm{n}^{\circ}$ 2006/022, les ressorts des Tribunaux administratifs. En effet, l'article 5 de la loi $\mathrm{n}^{\circ}$ 2006/022, considérée comme le repère des initiateurs du décret, précité, ne faisait aucune précision sur les ressorts desdites juridictions. Néanmoins, notons que l'alinéa 2 de cet article 5 ne faisait allusion au ressort que lorsqu'il dispose que : "toutefois, suivant les nécessités de service, le ressort d'un Tribunal administratif peut être, par décret du Président de la République, étendu à plusieurs régions ». La charge revenait alors à ce nouvel instrument juridique de désigner les ressorts correspondants. À cet effet, ce texte désigne les différentes régions comme étant les ressorts desdits Tribunaux. Par exemple, la région du Nord-Ouest constitue le ressort du Tribunal administratif de Bamenda dont le siège se trouve à Bamenda, chef-lieu de ladite région.

Pour comprendre la démarche du législateur il faudrait peut-être se référer à deux éléments : dans un premier temps, il faut partir de la définition même de la notion de ressort. Ce vocable désigne, selon un auteur, à la fois, l'étendue de la compétence géographique d'une juridiction, les valeurs à l'intérieur desquelles elle peut statuer et à l'intérieur de ces valeurs, les sommes au-delà desquelles les jugements qu'elle prononce sont susceptibles d'appel. D'un point de vue géographique, poursuit l'auteur, le ressort est la partie du territoire national sur l'étendue duquel s'exerce la compétence d'une juridiction ${ }^{64}$. Autrement dit, il correspond à la compétence dite « rationne loci » de la juridiction. Cette dernière

64 Voir S. Braudo, Dictionnaire de droit privé, disponible sur le sitehttp://www.dictionnairejuridique. com/definition/ressort.php. 
branche de la définition permet de comprendre la position du législateur camerounais. En effet, pour lui, il fallait rattacher chaque Tribunal administratif, sur le plan géographique, à l'ensemble de la région concernée.

Dans un second temps, et c'est le second argument qui permet de s'en convaincre, le décret précité s'est appuyé sur l'organisation des juridictions de l'ordre judiciaire au sein duquel les juridictions d'appels sont reparties en fonction des régions; leurs ressorts étant constitués par l'ensemble de la région concernée. L'article 19 de la loi n 2006/015 du 29 décembre 2006 portant organisation judiciaire en est une bonne illustration. On note à ce sujet que la première colonne du tableau dressé à l'article 2 du décret du 15 mars 2012, récapitulant les sièges et ressorts des Tribunaux administratifs, est intitulée : "Cour d'appel».

Cette répartition pourrait poser moins des conflits de compétence ${ }^{65}$ entre les différents Tribunaux administratifs. Ainsi, parmi les apports du décret du 15 mars 2012, on peut noter aussi que le législateur a bien pris en compte les éventuels conflits de compétence « rationne loci » qui pouvaient survenir entre les différents Tribunaux administratifs. Pour cela, il a arrimé la répartition des Tribunaux administratifs aux dispositions de la loi n 2006/022 qui dispose, d'une part, notamment en son article 15, que le Tribunal administratif territorialement compétent est, soit, celui dans le ressort duquel a légalement son siège l'autorité qui a pris la décision attaquée, soit, de la résidence du demandeur, soit, de la situation des biens, du lieu d'exécution du contrat, du fait dommageable si ce fait est imputable à une décision. D'autre part, cette même disposition précise qu'est compétent, toujours du point de vue territorial, le Tribunal administratif du siège de l'autorité ayant pris la décision attaquée; qui l'est également pour connaître de l'action en indemnité imputable à la décision querellée, pour des recours en interprétation et appréciation de la légalité de l'acte litigieux intervenant sur renvoi de l'autorité judiciaire.

Par ailleurs, eu égard à l'article 16 de la loi n²006/022, est compétent, territorialement, le Tribunal administratif qui l'est aussi territorialement pour connaître d'une demande principale; il l'est également pour toute demande accessoire, incidente ou reconventionnelle relevant de la compétence des Tribunaux administratifs.

En raison de cette répartition de la compétence territoriale des Tribunaux administratifs, le décret de 2012, en attribuant à chaque région un Tribunal et sans inclure des « Tribunaux administratifs interrégionaux » a évité les éventuels conflits de compétence. Ainsi, chaque Tribunal est compétent, territorialement, en fonction de son ressort bien délimité. Cette répartition spatiale des juridictions administratives correspond sans doute à une justice de proximité entre ces institutions chargées d'assurer le contrôle juridictionnel de l'administra-

65 Au sujet de la complexité de la répartition des compétences juridictionnelles, voir : S. V. Ntonga Bomba, « La répartition des compétences en matière administrative au Cameroun de 1972 à 2009 », Cahiers Juridique et Politiques, Faculté des Sciences Juridiques et Politiques de l'Université de Ngaoundéré, 2009, pp. 303-310; B.-R. Guimdo Dongmo, « Le droit d'accès à la justice administrative au Cameroun. Contribution à l'étude d'un droit fondamental », op. cit, pp. 494 et s. 
tion et les administrés. Cependant, cette justice administrative de proximité paraît insuffisante à plusieurs égards.

\section{B. L'insuffisante justice de proximité par la répartition spatiale quasi-centralisée des juridictions administratives}

$\mathrm{Au}$ Cameroun, compte tenu de la nouvelle répartition des juridictions administratives, l'idée de la justice administrative de proximité reste limitée. Cette limitation est liée non seulement de l'absence de délocalisation des juridictions administratives suprêmes (I), mais aussi d'une délocalisation inachevée des juridictions de premier ressort (II).

\section{Une insuffisante justice de proximité par l'absence de délocalisation des juridictions administratives suprêmes}

Les juridictions administratives dites suprêmes, suprématie par rapport aux juridictions de premiers ressorts, n'ont pas été géographiquement rapprochées des justiciables. Il s'agit, d'une part, de la juridiction chargée de connaître des pourvois en cassation (1), et, d'autre part, de celle qui connaît des appels (2).

\section{La traditionnelle centralisation de la juridiction administrative de cassation}

Il faut préciser que les juridictions administratives suprêmes ici s'entendent comme les instances juridictionnelles du contentieux administratif qui se situent à un niveau autre que les juridictions de premiers ressorts. Le caractère suprême s'apprécie donc ici par rapport aux Tribunaux administratifs qui sont des juridictions d'instances. Il s'agit du double degré de juridiction qu'est l'appel et la juridiction suprême proprement dite qu'est la cassation.

En matière de pourvoi en cassation, c'est la Chambre administrative qui en assure l'office. Il convient de rappeler que jusqu'à la réforme de 2006, ladite Chambre faisait office de juridiction de jugement et d'appel. Désormais, elle est érigée en juridiction d'appel et de cassation. Autrement dit, jusqu'en 2006, l'appel représentait la voie d'achèvement du contentieux administratif au Cameroun.

Sur le positionnement spatial de cette institution, sa centralisation relève de l'organisation classique des ordres de juridictions. En effet, en matière de pourvoi en cassation, on ne saurait avoir une diversité de juridiction dans la mesure où, une Cour suprême a pour rôle, en tant que juridiction de cassation, de veiller à la bonne application de la loi. En contentieux administratif, comme dans l'ordre judiciaire, cette Cour n'a pas pour mission de statuer comme un troisième degré de juridiction. Sa mission est de veiller à la conformité des jugements aux lois, à la bonne mise en œuvre de celles-ci par les autres juridictions que sont les Tribunaux administratifs et les Cours d'appel. Elle est donc la gardienne d'une application uniforme des lois en vigueur sur l'ensemble du territoire national. La Chambre administrative de la Cour suprême au Cameroun apparaît ainsi comme le Conseil d'État en France, donc en tant que juge suprême de «l'ensemble des litiges qui relèvent de la compé- 
tence de la juridiction administrative dans son ensemble ». ${ }^{66}$ Cela explique certainement pourquoi elle ne peut qu'être unique donc géographiquement située dans la localité siège des institutions du pays.

C'est ce qu'illustre l'ensemble des dispositions, constitutionnelles et législatives, en la matière. D'une part, selon l'article 38 de la Constitution camerounaise, "la Cour suprême est la plus haute juridiction de l'Etat en matière [...] administrative [...] ». D'autre part, l'unicité du ressort territorial de cette juridiction est fixée par l'article 3 de la loi $n^{\circ}$ 2006/016 sur le fonctionnement de la Cour suprême qui dispose que «le siège de la Cour suprême est à Yaoundé, et son ressort s'étend sur l'ensemble du territoire national ».

Du point de vue de sa compétence, en tant que juridiction de cassation, cette compétence pouvant expliquer sa position géographique et son unicité, il suffit de rappeler les dispositions de la loi $\mathrm{n}^{\circ}$ 2006/016, précitée, notamment en son article 9 alinéa 2, qui dispose que «chaque section" de la Chambre administrative « connaît $[. .$.$] des pourvois en cassa-$ tion relatifs aux matières qui relèvent de sa compétence ». S'agissant de ce pourvoi, l'article 35 de la même loi rappelle que les cas d'ouverture du pourvoi sont : «

a) l'incompétence;

b) la dénaturation des faits de la cause ou des pièces de la procédure;

c) le défaut, la contradiction ou l'insuffisance de motifs,

d) le vice de forme; [...]

e) la violation de la loi;

f) la non-réponse aux conclusions des parties ou aux réquisitions du Ministèrepublic;

g) le détournement de pouvoir;

h) la violation d'un principe général de droit;

i) le non-respect de la jurisprudence de la Cour suprême ayant statué en sections réunies d'une Chambre ou en Chambres réunies ».

Ainsi, ce n'est que dans ces différents cas que la Chambre administrative est appelée à se prononcer, bien entendu, sur le plan juridictionnel. Il est également important de rappeler les dispositions de la loi n²006/022, précitée, en son article 116, qui prévoit que «les décisions rendues en premier et dernier ressort par le Tribunal administratif sont susceptibles de pourvoi devant la Chambre administrative [...] ». Par ailleurs, en dehors du pourvoi, l'article 40 de la Constitution prévoit également que cette Chambre peut aussi connaître «[...] de toute autre matière qui lui est expressément attribuée par la loi ». Tous ces éléments traduisent le caractère suprême et unique de cette institution ainsi que la situation géographique, centralisée, qui est la sienne. Ainsi, cette absence de proximité n'est pas fondamentalement contestable. En revanche, par rapport à l'instance d'appel, la centralisation de celle-ci au sein de la Chambre administrative de la Cour suprême est critiquable car elle rend difficile l'accès au juge.

66 Y. Robineau, D. Truchet, Le conseil d'Etat, Paris, Puf, col. « Que sais-je », 2e, 2002, p. 70; B. Odent, D. Truchet, La justice administrative, Paris, Puf, col. « Que sais-je », 2e , 2008, p. 27. 
2. La centralisation de la Chambre administrative de la Cour suprême au titre de juridiction d'appel

La nouvelle répartition spatiale des juridictions administratives au Cameroun, avec notamment l'absence de déconcentration de la juridiction d'appel, apparaît inéquitable et même illégale (a). Pourtant, l'examen de la jurisprudence de la Chambre administrative montre que l'idée d'une proximité entre cette juridiction et les justiciables est nécessaire (b).

a. L'insuffisante justice de proximité par l'exercice exclusive de l'appel au sein de la Chambre administrative de la Cour suprême

L'on ne saurait nier que les décrets de 2012, précités, ont rendu effective la nouvelle répartition spatiale de la justice administrative au Cameroun. Celle-ci se caractérise désormais par une certaine proximité par rapport aux justiciables. Mais cette proximité ne concerne pas l'appel. Jusqu'à l'adoption des lois de 2006, il existait une seule instance statuant en appel à savoir la Chambre administrative de la Cour suprême. Les juridictions n'étaient pas réparties sur l'ensemble du territoire comme cela s'observe dans l'ordre judiciaire.

Il est important de rappeler qu'au sein d'une organisation juridictionnelle classique, l'appel traduit ce qu'on nomme communément le double degré de juridiction. Il permet de juger une même affaire par deux instances juridictionnelles différentes, notamment en cas d'insatisfaction d'une partie face au jugement rendu par un premier juge qu'on désigne généralement juge d'instance ou de premier ressort. Un tel système apparaît intéressant dans la mesure où il traduit certainement le souci d'une bonne administration de la justice. ${ }^{67}$ En effet, cette possibilité offerte aux parties à un procès, lorsqu'elles éprouvent de l'insatisfaction sur la décision rendue par une juridiction de première instance, de solliciter une autre juridiction, supérieure à la première, afin que l'affaire soit rejugée, est une voie de recours.

Dans le contexte camerounais, l'absence de proximité se traduit ici par le fait que ce double degré de juridiction est assuré uniquement par la Chambre administrative de la Cour suprême. En effet, selon l'article 2 de la loi nº 2006/016 du 29 décembre 2006 portant organisation et fonctionnement de ladite Cour, cette dernière « [...] est la plus haute juridiction de l'Etat en matière judiciaire, administrative et des comptes ». L'article 3 du même texte précise que : "le siège de la Cour suprême est à Yaoundé » et « son ressort s'étend sur l'ensemble du territoire national ». L'article 7 ajoute, par ailleurs, que « la Cour suprême comprend :

- une Chambre judiciaire;

- une Chambre administrative;

- une Chambre des comptes $[\ldots] »$;

67 R. Vandermeeren, « Appel : introduction de l'appel », Dalloz, Répertoire de contentieux administratif, avril 2019, $\mathrm{n}^{\circ} 1$. 
Cette Chambre administrative est elle-même composée, comme le précise l'article 9 alinéa $1, \ll d '$

- une section du contentieux de la fonction publique;

- une section du contentieux des affaires foncières et domaniales;

- une section du contentieux fiscal et financier;

- une section du contentieux des contrats administratifs;

- une section du contentieux de l'annulation et des questions diverses ».

Selon l'alinéa 2 de cet article 9, « chaque section connaît des appels et des pourvois en cassation relatifs aux matières qui relèvent de sa compétence ».

Il convient de souligner également que l'article 38 de la même loi dispose que « la Chambre administrative est compétente pour connaître [...] des appels formés contre les décisions rendues en matière de contentieux des élections régionales et municipales [...]».

Toutes ces dispositions relatives au double degré de juridiction qu'assure la Chambre administrative comportent également un fondement constitutionnel. Ce fondement réside en l'article 40 de la Constitution qui dispose que « la Chambre administrative connaît [...] en appel du contentieux des élections régionales et municipales. Elle statue souverainement sur les décisions rendues en dernier ressort par les juridictions inférieures en matière de contentieux administratif [...] ». L'analyse de ces dispositions montre, en guise de droit comparé, que contrairement à la réforme opérée en France avec la loi du 31 décembre 1987 qui avait créé les Cours administratives d'appel, limitant ainsi la compétence du Conseil d'État à la seule mission de cassation, ${ }^{68}$ du moins sur le plan de ses compétences juridictionnelles, le législateur camerounais n'a pas songé à répartir cette compétence sur l'ensemble du territoire national. La Chambre administrative de la Cour suprême poursuit donc, comme auparavant, son rôle de juridiction d'appel. Cela implique, d'une part, un alourdissement des charges de la juridiction supposée se limiter au rôle d'une juridiction suprême. Il en est ainsi parce que « chaque section connaît des appels et des pourvois en cassation relatifs aux matières qui relèvent de sa compétence ». D'autre part, cela entraîne une absence de proximité physique entre les justiciables et cette juridiction. Ainsi, le double degré de juridiction administratif camerounais n'a pas complément été réformé.

En d'autres termes, en droit camerounais, depuis la réforme de 2006, l'appel est assuré non pas par des Cours administratives d'appel réparties sur l'ensemble du territoire national mais uniquement par la Chambre administrative de la Cour suprême. Ladite Chambre dont le siège se trouve exclusivement à Yaoundé devient non seulement juridiction d'appel, mais aussi juridiction de cassation.

Cela dit, en tant que juridiction statuant en appel, l'on doit relever qu'elle est la seule en la matière. Ce qui pose le problème de son éloignement. Cette absence totale des juridictions d'appel dans les régions du pays pose un problème sérieux quant à l'accès au juge. 
Par ailleurs, on peut même soutenir que cette répartition spatiale est illégale. En effet, selon l'article 37 alinéas 1 et 2 de la Constitution, « la justice est rendue sur le territoire de la République au nom du peuple camerounais », d'une part, et, d'autre part, ce "pouvoir judiciaire », qui selon cette même Constitution est «indépendant du pouvoir exécutif et $d u$ pouvoir législatif», est "exercé par la Cour suprême, les Cours d'appel, les Tribunaux». Cette disposition est reprise et détaillée par la loi n 2006/015 du 29 décembre 2006 portant organisation judiciaire, notamment en son article 3 qui dispose que «l'organisation judiciaire comprend:

- la Cour suprême;

- les Cours d'appel;

- les Juridictions inférieures en matière de contentieux administratif [...]».

Cette nomenclature montre bien que le constituant n'a pas seulement consacré une Cour d'appel mais plusieurs. Le vocable «les Cours d'appel » ne saurait être logiquement interprété comme exclusif d'une diversité des juridictions statuant en appel dans l'ordre administratif. Il ne semble donc pas excessif de parler d'une illégalité puisque cette disposition parle d'une diversité des Cours d'appel. Comment donc comprendre que dans l'ordre judiciaire lesdites Cours soient au nombre de dix mais pas plus d'une seule dans l'ordre administratif? Cette limitation peut certainement s'expliquer par les restrictions financières.

Sur la question de la proximité géographique, eu égard à cette centralisation, l'accès au juge reste difficile. Cette difficulté s'apprécie également par rapport au contexte. En effet, il faut rappeler que la moyenne, par exemple, de la distance qui sépare Yaoundé, siège de la Cour suprême, de la partie septentrionale du pays est d'environ mille kilomètres. Cette distance est importante compte tenu non seulement des difficultés liées au transport mais aussi des moyens de communication. Cette situation tient donc encore, géographiquement, le justiciable camerounais loin du juge administratif.

Pourtant, et cela paraît tout à fait logique, l'on devrait avoir autant de juridictions d'appel qu'il y a de Tribunaux administratifs par région sur l'ensemble du territoire. Cela est important car, d'une part, avec l'adoption d'un nouveau code de la décentralisation au Cameroun, l'action du juge administratif trouve encore toute son importance. D'autre part, selon la Charte africaine sur les valeurs et les principes du service public et de l'administration, ratifiée le 7 septembre 2014 par le Cameroun, il est fait obligation à l'administration, non seulement, d'être « organisée de manière à assurer et faciliter l'accès aux prestations de service public adéquates », mais aussi et surtout de façon à « s'assurer que les services sont fournis au plus près des usagers $\gg .{ }^{69}$

De ce point de vue, même si cela n'aurait résolu le problème qu'à un seul niveau, le législateur aurait pu mettre en place des Cours d'appel administratives dans les régions du pays où la distance et la fracture numérique sont importantes par rapport au siège de la Cour suprême. En effet, la justice, en général, et administrative en particulier, « doit aussi 
être un service public de proximité, garantissant à tous- et notamment aux justiciables se trouvant dans une situation de fragilité -d'avoir recours à lui sans difficulté $» .{ }^{70}$ Cela est notable au Cameroun car, comme ont pu le relever de nombreux observateurs, si en «Europe occidentale, l'administration est le produit continu d'une complexification croissante et erratique de tout ou partie de la société, en Afrique francophone elle n'est le plus souvent qu'un appareil formel d'exploitation et de gestion. L'administration n'apparaît alors jamais comme l'instrument né de la complexification des structures économiques et sociales, mais comme un produit fini, préfabriqué, imposé pour assurer une succession d'État ». ${ }^{71}$ On sait également que les efforts de décentralisation n'ont pas toujours été « efficaces et l'organisation administrative contentieuse » étant calquée sur cette organisation globale de l'Etat, sa caractéristique principale est «l'éloignement de la justice des justiciables ». ${ }^{72}$ C'est bien le cas au Cameroun avec ce maintien de la Chambre administrative comme l'unique juridiction administrative d'appel. Dans ce cas, l'idée d'une justice administrative de proximité, d'un point de vue géographique, n'est pas soutenable.

\section{b. Une absence de proximité contraire à la jurisprudence}

Comme on vient de le montrer, dans le cadre du rapprochement géographique des juridictions administratives des justiciables au Cameroun, les juridictions administratives d'appel ne sont pas concernées. Or, d'un point de vue jurisprudentiel, l'idée d'une justice administrative de proximité avait déjà été implicitement relevée à l'occasion d'une instance en appel. C'est dire que la proximité judiciaire en contentieux administratif camerounais ne relève pas exclusivement des textes précités. Celle-ci avait déjà été soulignée dans une certaine mesure par la Chambre administrative de la Cour suprême dans l'affaire Albert Ono Ngafor.

Dans cette cause, la Chambre administrative de la Cour suprême va juger recevable l'appel interjeté par l'État du Cameroun le 14 août 1985, appel introduit par le biais du greffe du Tribunal de première instance de Ngaoundéré, contre la décision rendue en premier ressort le 14 juin 1984 dans l'affaire l'opposant à Monsieur Albert Ono Ngafor. Dans les faits, par un arrêté du 19 janvier 1981, le Préfet du département de la Mézam avait ordonné la destruction des clôtures et portail du collège "Macho Comprehensive High School » dont Monsieur Albert Ono Ngafor était responsable. Celui-ci va introduire un recours en annulation, y compris le paiement des dommages et intérêts, contre cette décision devant la Chambre administrative de la Cour suprême. La juridiction saisie avait fait droit à la requête du demandeur. C'est donc contre ce jugement que l'État du Cameroun va interje-

70 N. Braconnay, La justice et les institutions juridictionnelles, Paris, La documentation française, col. «Découverte de la vie publique », $3^{\mathrm{e}} \mathrm{ed}, 2019$, p. 33.

71 D. Darbon, Le paradoxe administratif : perspective comparée autour de cas africains, Thèse, Université de Bordeaux 1, 1991, p. 79.

72 A. Kah, La codification administrative au Sénégal, Thèse, Université Paris nord, 1996, pp. $277-$ 278. 
ter appel. Il est important de préciser que selon l'article 28 alinéa 1 de la loi n $75 / 17$ du 8 décembre 1975 qui organisait la procédure devant la Chambre administrative de la Cour suprême, la déclaration d'appel devait être faite au greffe de ladite Cour.

Ainsi, en recevant un recours exercé par le canal du service des greffes d'une juridiction de l'ordre judiciaire située dans une autre localité que celle de la juridiction compétente, le juge avait pris en compte cet éloignement géographique qui caractérise cette juridiction. Autrement dit, le juge administratif avait donné la possibilité à des justiciables qui résident hors de Yaoundé, siège de la juridiction, d'interjeter appel par le biais des juridictions du lieu où ils se trouvent.

Cette jurisprudence a donc essayé, non seulement, de rapprocher la justice du justiciable, mais aussi de permettre aux justiciables de faire face aux problèmes financiers, ambiants, lesquels sont liés aux déplacements vers la localité où se trouve cette juridiction. ${ }^{73}$ Il s'agissait ainsi, d'une solution qui met en exergue la nécessité de procéder à une déconcentration de la justice administrative au Cameroun. Le juge, pouvait-on dire, avait proposé au législateur une solution face à cet éloignement de la juridiction administrative camerounaise de ses justiciables. L'idée de la déconcentration de la justice administrative qui apparaît dans cette jurisprudence semble avoir finalement incité le législateur à procéder à une nouvelle répartition géographique des juridictions quelques années plus tard. Mais celle-ci n'est restée applicable qu'aux juridictions d'instances, donc elle a maintenu l'appel dans son statut d'antan à savoir celui d'une juridiction centralisée. Par ailleurs, même si les juridictions de premier ressort ont été déconcentrées, celle-ci demeure relative.

\section{L'insuffisante justice de proximité par un positionnement spatial quasi-centralisé des Tribunaux administratifs régionaux}

Il est important de rappeler dans un premier temps la compétence des Tribunaux administratifs. En effet, ces Tribunaux sont, selon l'article 2 de la loi $n^{\circ}$ 2006/022, précitée, des « juridictions inférieures en matière de contentieux administratif au sens de l'article 40 de la Constitution ». Ils « connaissent en premier ressort, du contentieux des élections régionales et municipales et en dernier ressort, de l'ensemble du contentieux administratif concernant l'Etat, les collectivités publiques territoriales décentralisées et les établissements publics administratifs [...]». Et en vertu de l'article 14 de la même loi, ils «sont, sauf dispositions contraires de la loi, juges de droit commun du contentieux administratif en premier ressort $»$. C'est dire toute l'importance de telles juridictions dans l'ordre administratif camerounais. Elles sont les juridictions les plus proches, « a priori », des justiciables. Elles traduisent certainement, sur le plan spatial, une justice administrative de proximité.

73 C. Keutcha Tchapnga, « Notes sous CS/CA, arrêt du 16 août 1990, Albert Ono Ngafor c/ Etat du Cameroun (MINAT) », Revue électronique Afrilex, $\mathrm{n}^{\circ}$ 5, pp. 269-285. Disponible sur le sitehttp//w ww.afrilex.u-bordeaux 4.fr. 
A l'analyse, il faut souligner que même si aujourd'hui, avec la création de ces juridictions administratives régionaux, le justiciable qui réside dans la commune de Batouri a son juge à Bertoua, donc plus à Yaoundé, ${ }^{74}$ le problème de l'accessibilité au juge administratif, sur le plan géographique, n'est pas suffisamment résolu. On ne peut pas méconnaître que le justiciable qui part de la commune de Deuk, de Bokito ou de Ngambé-Tikar, par exemple, devra parcourir plusieurs kilomètres pour atteindre Yaoundé le siège du Tribunal administratif régional compétent. En comparant cette organisation avec celle des juridictions de l'ordre judiciaire, on note une différence importante du point de vue de la proximité géographique.

Pourquoi le législateur n'adopte-t-il pas la même démarche pour les juridictions d'instances de l'ordre administratif? Comme l'a d'ailleurs suggéré un observateur, pourquoi ne confie-t-il pas, par souci de proximité géographique, à certaines juridictions inférieures de l'ordre judiciaire, le soin de statuer sur les recours qui devraient être portés devant les Tribunaux administratifs régionaux ? $^{75}$ Mais cette solution ne devrait s'appliquer qu'aux justiciables qui se trouvent dans des circonscriptions administratives très éloignées de leur Tribunal administratif. L'on pourrait aussi, à titre de droit comparé, proposer la création des sections administratives au sein des Tribunaux de l'ordre judiciaire comme l'avait fait le législateur gabonais. ${ }^{76}$

En l'état actuel, on peut relever que même si la réforme de 2006 a réduit la distance par rapport à Yaoundé, le siège de la Chambre administrative de la Cour suprême, le justiciable qui réside, par exemple, dans la localité de Foumban devra parcourir plus de soixante-dix kilomètres pour atteindre la localité de Bafoussam, siège de la juridiction administrative territorialement compétente. ${ }^{77}$

74 Située à Yaoundé, la juridiction administrative se trouvait à plus de $200 \mathrm{~km}$ de la plupart des grandes villes du pays.

75 A.-D. Olinga, Le nouvel environnement juridique et institutionnel des élections au Cameroun, Yaoundé, Presses universitaires d'Afrique, 2007, pp. 59 et s.

76 Certains auteurs estiment, cependant, que la centralisation et l'unité sont les premières conditions du succès d'une juridiction car, le cloisonnement territorial des autorités judiciaires et des services de police entraîne la multiplicité des procédures, la disharmonie des sentences rendues et finalement l'inefficacité de la répression. Au Cameroun, le professeur Henri Jacquot justifiait cet état de choses par des raisons de simplicité et d'économies de moyens. Voir respectivement : A. Vitu, "Une nouvelle juridiction d'exception : la cour de sûreté de l'État ", Revue des Sciences Criminelles, 1994, p.1, cité par A. MinkoaShe, Droit de l'homme et droit pénal au Cameroun, Paris, Economica, coll. « La vie du droit en Afrique », 1999, p. 252.

77 La loi prévoit, en revanche, que «si les parties ou l'une d'elles sont dans l'impossibilité de comparaître, le Tribunal peut commettre un de ses juges qui se transporte auprès d'elle accompagné par le greffier. En cas d'éloignement des parties ou de l'une d'elles rendant le déplacement difficile ou onéreux, la juridiction saisie peut donner commission rogatoire au tribunal de leur domicile ou de leur résidence, pour les entendre ensemble ou séparément, suivant les circonstances ». Mais il s'agit d'une mesure exceptionnelle, celle-ci ne saurait sérieusement résoudre le problème du rapprochement de la juridiction administrative de ses justiciables. Art. 68, 78, loi n ${ }^{\circ}$ 2006/022, relative aux Tribunaux administratifs, précitée. 
Par ailleurs, d'après l'article 5 alinéa 2de la loi n²006/022, précitée, « suivant les nécessités de service, le ressort d'un Tribunal administratif peut être, par décret du président de la République, étendu à plusieurs régions ». Cette disposition consacre donc la mise en place possible des Tribunaux administratifs interrégionaux. Cela donne la possibilité de réduire davantage le rapprochement géographique entre ces juridictions et les justiciables. Par exemple, si le Tribunal administratif de la région du Centre voyait son ressort étendu à la région de l'Adamaoua, le justiciable qui réside à Ngaoundéré se trouve à une distance de plus de huit cents kilomètres de son siège. De ce point de vue, le rapprochement de la justice administrative camerounaise des justiciables n'est pas suffisamment assuré. Il est cependant intéressant de voir que le décret du 15 mars 2012 n'a pas mis en place des «Tribunaux administratifs interrégionaux ».

Dans un pays où, comme dans la majorité des pays d'Afrique francophone, "la maxime nul n'est censé ignorer la loi est très contestée à cause de l'absence d'une véritable politique de publication et de diffusion des règles juridiques », où «la pratique du journal officiel est très limitée $»,{ }^{78}$ il convient de corriger une telle répartition spatiale des juridictions administratives afin d'assurer une meilleure proximité physique entre ces juridictions et les justiciables.

Comment y parvenir? En dehors des solutions précédemment envisagées, le renforcement du rapprochement spatial de la justice administrative des administrés peut aussi passer par la mise en place des audiences foraines. Cette dernière solution est consacrée dans des pays comme la Guinée. ${ }^{79}$ Cela est bien envisageable au Cameroun car, comme le souligne un observateur, "la notion de proximité judiciaire s'analyse aussi selon deux modes distincts de fonctionnement » à savoir : l'usage « d'un personnel sédentaire ou ambulatoire $\gg{ }^{80}$

Ainsi, la proximité entre les justiciables et les Tribunaux administratifs n'est pas confortable au Cameroun. Vu le contexte, à savoir : le décalage entre campagnes et villes, qui est tout à fait naturel, mais dont la disparité est très accentuée eu égard aux héritages de la colonisation, la proximité physique avec ces juridictions reste limitée. ${ }^{81}$ Et en dépit de quelques efforts de développement menés depuis les indépendances, la situation n'a pas significativement changé. Ainsi, comme le relève fort justement le professeur Amadou Kah, «l'hypertrophie des grandes villes », héritage colonial, n'a pas été suffisamment résolue en

78 G. Fouda, «L'accès au droit : richesse et fécondité d'un principe pour la socialisation juridique et 1'Etat de droit en Afrique Noire francophone ", Afrilex, 2000/01, p. 4.

79 H. I. Diallo, La réforme de la justice guinéenne. Défis et stratégie, Paris, L'Harmattan, 2011, préface de Mamadou Alioune, p. 50.

80 M. Charageat, « Marie Houllemare et Diane Roussel (dir), Les justices locales et les justiciables. La proximité judiciaire en France du Moyen Âge à l'époque moderne », Criminocorpus [En ligne], Années antérieures, 2016, mis en ligne le 27 janvier 2016, consulté le 22 février 2020. URL : http:/ /journals.openedition.org/criminocorpus/3139.

81 A. Kah, La codification administrative au Sénégal, op. cit, ibid. 
raison des « choix de décentralisation surveillée ». ${ }^{82}$ De ce point de vue, la justice administrative camerounaise, à plusieurs égards, reste encore géographiquement éloignée des justiciables. La justice administrative dite de proximité, reste donc encore relative en droit camerounais.

\section{CONCLUSION}

À la question de savoir si la justice administrative en Afrique francophone ne souffre plus de dysfonctionnements liés à son inégale répartition géographique, limitant sa proximité avec les justiciables, la réponse doit être nuancée. L'exemple du Cameroun, avec notamment sa nouvelle répartition spatiale des juridictions administratives, n'apporte pas une solution définitive à ce problème. En effet, malgré les progrès considérables qui ont été enregistrés, la mise en place d'une justice administrative dite de proximité, au plan géographique, laisse l'observateur perplexe. La question de la proximité n'est pas entièrement résolue car l'accès demeure difficile en raison des insuffisances du rapprochement opéré pour les juridictions inférieures, et son absence en ce qui concerne les autres juridictions comme notamment l'appel. En effet, au-delà de l'absence de proximité entre les justiciables et la juridiction d'appel, la proximité adoptée pour les juridictions inférieures ne prend pas en compte le contexte dans lequel ces juridictions se déploient.

Le problème est lié au fait que dans les pays africains, en général, et au Cameroun en particulier, la fracture numérique est considérable. Pourtant, dans le cadre des politiques de promotions technologiques actuelles, un accent particulier est mis sur ces «technologies comme vecteurs de modernisation du fonctionnement des services publics et de rapprochement avec des citoyens $»{ }^{83} \mathrm{C}$ 'est de façon très progressive que la plupart des pays africains s'adaptent à ce nouvel outil de travail. ${ }^{84}$ Les moyens de communication sont également insuffisants. $^{85}$

Au Cameroun, compte tenu de l'insuffisance des travaux d'entretien, les routes sont très exposées aux intempéries et donc souvent impraticables. Les voies ferrées et aéroportuaires ne couvrent que quelques régions. Il en existe sept notamment Douala, Yaoundé-Nsimalen, Maroua-Salak, Bamenda, Bertoua et Ngaoundéré. ${ }^{86}$ Au-delà de ce nombre réduit, les difficultés financières ne permettent pas à de nombreux citoyens d'en faire usage. L'usage des

82 Ibid.

83 L. Dumoulin, C. Licoppe, « Technologie, droit et justice : quelques éléments de mise en perspective », Revue Droit et cultures, ${ }^{\circ}$ 61, 2011/1, p. 23.

84 Exemple récent en droit ivoirien : Art.31-2 (nouveau), Code de procédure pénale civile et administrative, introduit par l'ordonnance $\mathrm{n}^{\circ}$ 2015/180 du 24 mars 2015 .

$85 \mathrm{~K}$. Ahadzi, «L'accès à la justice. Brèves réflexions sur le cas des États d'Afrique noire francophone ", in M. Pâques et M. Faure (dir), La protection de l'enivrement au cœur du système juridique international et du droit interne. Acteurs, valeurs et efficacité, Bruxelles, Bruylant, 2003, p. 404.

86 Voir : http://adcsa.aero/aeroports. Consulté le 04 mars 2020. 
transports routier reste le moyen le plus utilisé. Dans tous les cas, ces moyens de communication ne sont pas très performants ${ }^{87}$ et ne peuvent pas convenablement accompagner la justice.

Une étude récente sur la question relève, par exemple, que le Cameroun « connaît un réel problème de connexion des zones frontalières aux centres nationaux du pays (Douala et Yaoundé) que ce soit en termes de routes, de télécommunications ou de signal radio-télévisuel [...]. Les infrastructures de transport existantes, faute d'entretien [...] se sont dégradées rapidement $\gg .88$

On peut ajouter à cela le contexte sécuritaire difficile en ce moment dans certaines régions du pays, comme le Nord-ouest et le Sud-ouest, lequel se traduit par la destruction de nombreuses infrastructures, y compris celles de la justice, par des bandes armées. Ainsi que le souligne le rapport de l'année 2017 du ministère de la justice sur l'état des droits de l'Homme au Cameroun, cette crise sécuritaire a «affecté le fonctionnement des services publics », en général, même si «dans le cas spécifique des juridictions, des dispositions ont été prises pour maintenir la continuité de leurs activités malgré la destruction par incendie de certains bâtiments abritant des services judiciaires $[\ldots] \gg .{ }^{89}$

Dans ces conditions, des aménagements plus importants semblent nécessaires pour assurer une proximité géographique suffisante entre le juge administratif et ses justiciables. Pour y parvenir, la solution peut être recherchée au niveau de la répartition de ces juridictions en les rapprochant davantage des justiciables par le biais des juridictions de l'ordre judiciaire, notamment dans les zones difficiles d'accès. La solution peut également être recherchée dans un cadre plus global. Ainsi que s'interrogeait le professeur Jean-Paul Markus à propos du droit administratif en Afrique, «ne peut-on pas imaginer une autre forme d'harmonie, basée non plus sur une structure centralisée, mais sur un polycentrisme juridique assumé, tenant également compte des pouvoirs traditionnels? $\gg .^{90}$

87 R. Mballa Owono, Les délais de distance en contentieux administratif camerounais, Mémoire de DEA, Université de Douala, 2004, p. 60.

88 A. Yemmafouo, Ako Y. Oneke, L. Uwizeyimana, «Infrastructures de transport et destin des territoires frontaliers du Sud-Ouest camerounais : cas de Mamfé et sa région ", Les Cahiers d'OutreMer [En ligne], 259 | Juillet-Septembre 2012, mis en ligne le 01 juillet 2015, consulté le 22 février 2020. URL : http://journals.openedition.org/com/6663; DOI : https://doi.org/10.4000/com.6663.

89 Rapport du Ministère de la Justice sur l'état des droits de l'Homme au Cameroun en 2017, p. 53. Disponible sur le sitehttp://minjustice.gov.cm/index.php/fr/missions/droits-de-1-homme/297-rappo rt-du-ministere-de-la-justice-sur-1-etat-des-droits-de-1-homme-au-cameroun. Consulté le 04 mars 2020;voirégalement : L. Esso, «En toute sécurité juridique et judiciaire! », Editorial, in, Justitia, Magazine d'informations générales du Ministère de la justice, $n^{\circ}$ 011, juin 2018, p. 5.

90 J.-P. Markus, « Le droit administratif africain à la recherche de ses propres modèles ", in M. Ondoa, P.-E. Abane Engolo (dir), Les transformations contemporaines du droit public en Afrique, Paris, L'Harmattan, 2018, p. 34. 